\title{
Reconstruction and identification of photons and electrons with the ATLAS detector
}

\author{
O. Fedin ${ }^{\mathrm{a}}$, On behalf of the ATLAS collaboration
}

${ }^{a}$ Petersburg Nuclear Physics Institute, St. Petersburg, 188300, Russia

\begin{abstract}
Excellent electron and photon reconstruction and identification capability will be needed over a broad energy range for most physics studies with the ATLAS detector at the Large Hadron Collider (LHC). Events with electrons and photons in the final state are important signatures to rediscover of many Standard Model (SM) processes (electroweak vector bosons, top quarks etc...), to search for new physics beyond the SM (new resonances at TeV scale, SUSY etc...), to discover of Higgs boson $(H \rightarrow 4 e, H \rightarrow \gamma \gamma)$ and to understand of detector performance and its calibration. This paper describes the overall ATLAS detector performance for the reconstruction and identification of photons and electrons over a wide range of transverse energies, spanning from a few $\mathrm{GeV}$ to $5 \mathrm{TeV}$.
\end{abstract}

Key words: electron, photon, identification, reconstruction, TRT, ATLAS, LHC

PACS: 29.40.Vj, 29.40.Gx, 29.90.+r

Physics performance requirements. With a total $p p$ inelastic cross section of order of $70 \mathrm{mb}$ at a center of mass energy of $14 \mathrm{TeV}$ at the LHC, a jet rejection factor exceeding $10^{5}$ ( $\sim 50$ times better than for Tevatron experiments) will be needed to extract a relatively pure inclusive electron signal in the moderate $p_{T}$ region between 20 and $50 \mathrm{GeV}$ from QCD jets faking electrons. The cross section of the SM Higgs boson decaying into two photons final state is small and a jet rejection factor of about 5000 should be reached to reduce the contamination of jets which contain a high fraction of photons from neutral hadron decays like $\pi^{0}$. In addition, the large amount of material in front of the electromagnetic calorimeters will deteriorate the performance of the electromagnetic calorimeter affecting the measured electron and photon energies and directions.

Detector description. The electromagnetic calorimeter and tracking system are involved for reconstruction and identification of electrons and photons with the ATLAS -detector [1]. The tracking system enclosed in a solenoidal magnet with $2 \mathrm{~T}$ field is composed of three subsystems: Pixel detector, Semi Conductor Tracker (SCT) and Transition Radiation Tracker (TRT). The Pixel detector and SCT are silicon detectors with a high granularity which provide a precise reconstruction of charged particles tracks and primary and secondary vertices. The TRT is a largescale gaseous detector which consists of thin-walled proportional drift tubes, called straw, filled by xenon based gas mixture. The TRT provides both continuous tracking in individual straws and electron identification using the capability to detect transition radiation X-ray photons

Email address: Oleg.Fedin@cern.ch (O. Fedin)

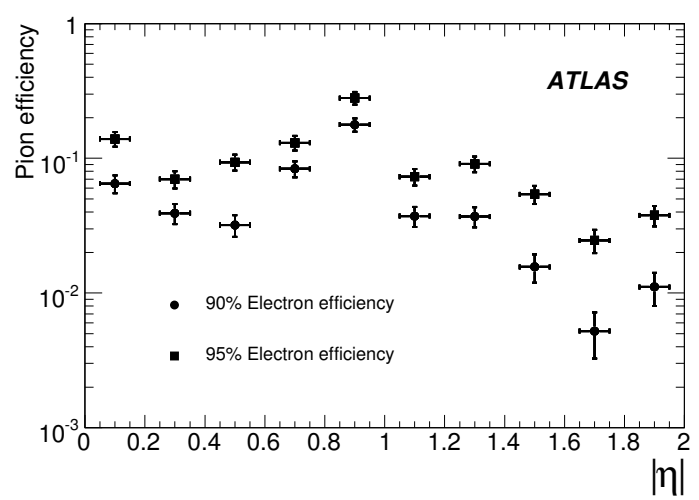

Figure 1: Pion efficiency expected from simulations as a function of $|\eta|$ for an efficiency of $90 \%$ or $95 \%$ for electrons with $p_{T}=25 \mathrm{GeV}$.

originating from thin foils between the straw layers. The TRT is important for overall momentum resolution, reconstruction of conversion electron and for particle identification. The TRT expected pion identification efficiency as a function of $\eta$ is shown in Fig. 1 for an electron efficiency of $90 \%$ or $95 \%$.

The ATLAS electromagnetic (EM) calorimeter is a $\mathrm{Pb}-$ LAr sampling calorimeter with accordion geometry for the absorbers and electrodes allowing for a full coverage of the azimuthal angle. The EM calorimeter is located after a solenoid which provides a $2 \mathrm{~T}$ magnetic field for track reconstruction by the ATLAS tracking system. The EM calorimeter is segmented in depth in three layers outwards from the beam axis. These layers are labeled "Strips", "Middle" and "Back". The total thickness of the calorimeter is greater than 22 radiation lengths $\left(\mathrm{X}_{0}\right)$. Up to $\eta=1.8$,

May 4, 2009 
the EM calorimeter is complemented by a thin presampler located in the calorimeter cryostat behind solenoid whose goal is to correct for the energy loss in the upstream material. The "Middle" layer contains most of the energy while the "Back" layer is used to correct longitudinal shower leakage. The "Strip" layer has a fine $\eta$ granularity in order to provide a precise measurement of the $\eta$ position of the incident particle and to allow an efficient $\gamma-\pi^{0}$ separation.

Track reconstruction. The ATLAS tracking system provides reconstruction of the charged particle tracks with $p_{T}>0.5 \mathrm{GeV}$ and pseudorapidity $|\eta|<2.5$. Two complementary algorithms are used to reconstruct tracks. The first tracking algorithm, called inside-out, starts with track seeds found in Pixel/SCT layers and extrapolates them into the TRT. The inside-out algorithm is efficient for tracks, coming from interaction point, i.e. for primary charged particles. The second algorithm, called back-tracking reconstructs tracks seeding from the TRT and extrapolates them to the SCT and Pixel. The track reconstruction efficiency for single electrons as a function of $|\eta|$ is shown in Fig. 2. The overall efficiency is a result of the merging of both algorithms. Efficiency curve reflects the shape of the amount of material in the tracking system and, as expected, becomes larger and more uniform at higher energies.

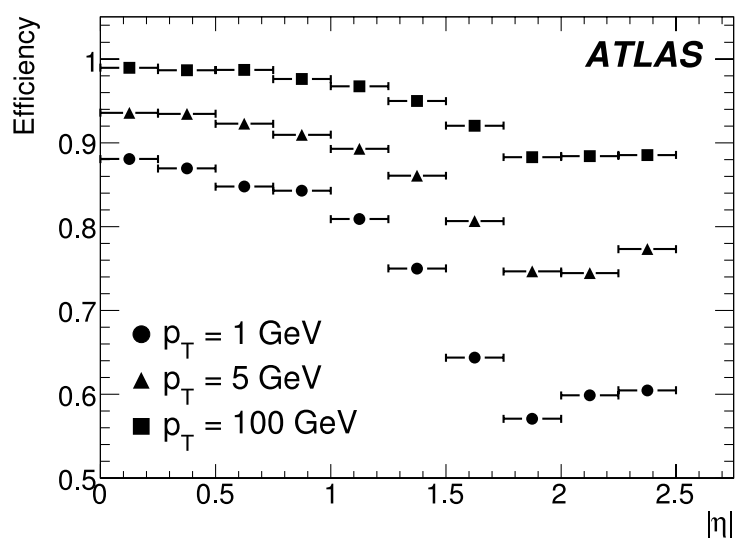

Figure 2: Track reconstruction efficiencies as a function of $|\eta|$ for electrons with $p_{T}=1,5$ and $100 \mathrm{GeV}$.

Conversion. Between 10 to $50 \%$ of photons have converted into electron-positron pairs before leaving the SCT at radii below $50 \mathrm{~cm}$. Therefore conversion reconstruction is very important to study of di-photon final states and to map of the material in the tracking system. At radii below $50 \mathrm{~cm}$ conversion vertices are found with an efficiency of about $80 \%$. At radii above $50 \mathrm{~cm}$ the efficiency drops, but the overall conversion efficiency can be greatly increased at large radii by flagging single TRT tracks as photon conversion using the capability of TRT detector to identify electrons and to reject pions.

Electron and photon reconstruction. The electron and photon reconstruction inside the ATLAS detector [2] starts from the energy measured in the calorimeter cells which is calibrated at the electromagnetic scale. A fixedsize rectangular cluster is built around a cell containing a local maximum in energy. The position of this rectangular window is chosen in order to maximize the amount of energy within the cluster. The cluster size is usually expressed in terms of the number of cells in the "Middle" layer (in pseudorapidity $\eta$ and azimuth $\varphi$ ) and at this reconstruction step the cluster size $5 \times 5$ is used. At the next step, for each cluster, the reconstruction algorithm performs a match with a track within a broad $\Delta \eta \times \Delta \varphi$ window with momentum compatible with the cluster energy $\left(\mathrm{E}_{\text {cluster }} / \mathrm{p}_{\text {track }}<10\right)$. If such a track is found, the reconstruction algorithm checks for the presence of an associated conversion. An electron candidate is created if a matched track is found and no conversion is flagged. Otherwise the candidate is classified as a photon. Depending on the particle hypothesis and calorimeter region, the cluster is then rebuilt with the appropriate size which is slightly different for electrons $(3 \times 7)$, photons $(3 \times 5)$ and converted photons $(5 \times 5)$. This early classification allows to apply different corrections to electron and photon candidates. The $\eta$ and $\varphi$ position of the cluster are computed as the energy-weighted barycenter of the cluster. The measured energy and position of the EM cluster are corrected at the cluster level taken into account the upstream material and the calorimeter segmentation (S-shape modulation for position, $\varphi$ modulation - due to accordion structure for energy). After these corrections, an $\eta$ position resolution of $\sim 3 \times 10^{-4}$ is achieved in the "Strip" compartment and of $\sim 6 \times 10^{-4}$ in the "Middle" layer. The $\varphi$ resolution is $0.5-1.5 \times 10^{-3}$. The energy of the cluster, which is the sum of the energies deposited by the shower in each layer and the presampler, is corrected for upstream energy loss and for lateral and longitudinal leakage. The correction factors are a function of the longitudinal barycenter of the shower and $\eta$ position of the cluster, but are energyindependent. The expected sampling (stochastic) term of the resolution for electron degrades from $9 \%-16 \%$ in the barrel, where material in front is minimal $\left(\sim 2.5 \mathrm{X}_{0}\right)$, to $23 \%$ in the transition region between barrel and end-cap, where material is maximal $\left(\sim 6 \mathrm{X}_{0}\right)$. The photons are less sensitive to the material and the expected sampling term of resolution is better than $12 \%$ over the whole $\eta$ range. The expected linearity, i.e. the ratio between reconstructed energy and the true particle energy, is better than $0.5 \%$ over whole $\eta$ range, both for electrons and photons over the energy range from $25 \mathrm{GeV}$ to $1 \mathrm{TeV}$.

Electron and photon identification. In order to identify electrons and photons and reject background from jets, a set of variables from calorimeter and tracker are used. The calorimeter variables exploit the precise measurement of the electromagnetic shower shape due to high lateral and longitudinal segmentation of the ATLAS EM calorimeter and the measurement of the fractional leakage. The track based cuts include track quality measurements, track to 
cluster matching constraints and TRT electron identification capability, which is defined as the ratio of the number of high threshold hits to the number of hits in TRT.

Both cut-based and multivariate discriminant techniques like the likelihood ratio, covariance matrix or neural network are used to identify isolated electrons and photons and to reject background from jets. The multivariate discriminant techniques give almost the same performance as the cut-based approach, but have the advantage to take into account correlations between variables.

For electrons, three different sets of cuts called loose, medium and tight are defined. The expected reconstruction and identification efficiency as a function of $\eta$ for three sets of cuts is shown in Fig. 3. The loose sets of cuts

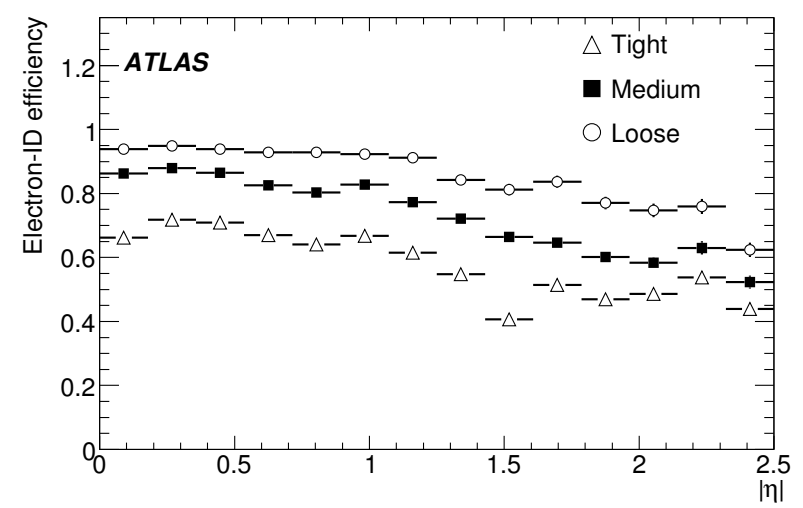

Figure 3: Electron identification efficiency versus pseudorapidity $|\eta|$ for different set of cuts.

use the fractional hadronic leakage and the shower shape variables in the "Middle" sampling. Loose set provides excellent identification efficiency of $\sim 88 \%$ for electrons, but low background rejection of $\sim 600$. The medium cuts use the "Strip" shower shape variables along with track variables which define the cluster matching and the track quality. It increases jet rejection up to 2000, while reducing the identification efficiency to $\sim 77 \%$. Tight cuts use all currently available particle identification variables for electrons. To reject electrons from photon conversion, some cuts are applied to the number of hits at Pixel detector. To reject the residual significant background from charged hadrons, TRT electron identification capability is used in the tight set. The tight selection provides the highest rejection against jets of $\sim 10^{5}$ along with reasonable electron identification efficiency of $\sim 64 \%$. Photon cut-based identification uses the calorimeter variables and additional track isolation cut, which is very powerful to reject jet background in both electron and photon identification. For photons, only one set of cuts is defined since the background from jets will be significant even after all avaiable cuts will be applied. The overall efficiency is above $80 \%$ and a jet rejection of about 8000 can be achieved for photons. The jet rejection strongly depends on parton type, gluon jets having a rejection better by a factor 10 .

The expected differential cross section after tight cuts (see Fig. 4) can be broken down into their three main components, isolated electrons from $W, Z$ and top-quark decays, non isolated electrons from b, c decays and residual jet background. The shapes of the spectra for nonisolated electrons and residual jet background are very similar, whereas the spectrum from isolated electrons exhibits the expected behaviour for a sample dominated by electrons from $W, Z$ decays. For an integrated luminosity of $100 \mathrm{pb}^{-1}$ we may expect approximately $10^{7}$ reconstructed and identified inclusive electrons from b, c decays with $\mathrm{E}_{T}>10 \mathrm{GeV}$, while for the same integrated luminosity we may expect only $5 \cdot 10^{5}$ such electrons with $\mathrm{E}_{T}>20 \mathrm{GeV}$ with a dominant contribution from $W, Z$ decay for $\mathrm{E}_{T}>35 \mathrm{GeV}$. These large data sample will clearly be extremely useful to certify many aspects of the electron identification performance of ATLAS with real data.

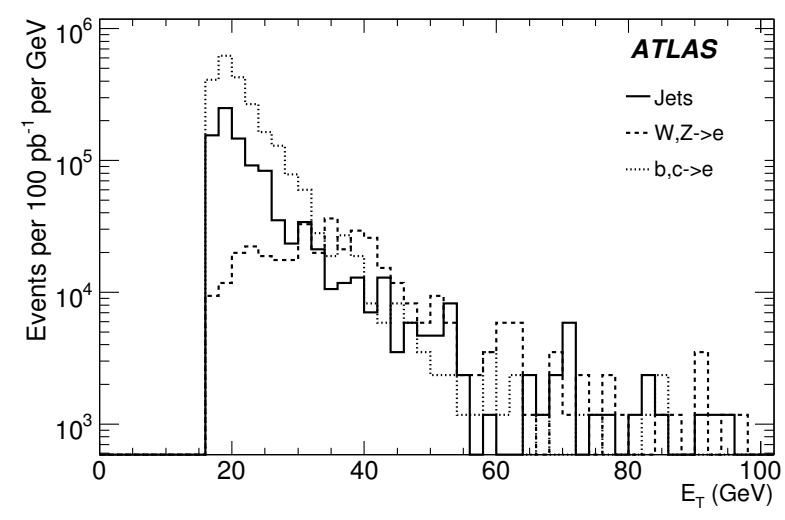

Figure 4: Differential cross-section as a function of $\mathrm{E}_{T}$ after tight cuts, shown separately for the expected components from isolated electrons, non-isolated electrons and residual jet background, for an integrated luminosity of $100 \mathrm{pb}^{-1}$ and for the simulated di-jet sample with $\mathrm{E}_{T}>17 \mathrm{GeV}$.

In-situ calibration. The measurement of the material distribution in front of calorimeter, the reconstruction and identification efficiency, the energy scale and the resolution from data itself are extremely important to tune the Monte-Carlo parameters to get the perfect simulation of the detector. The electron reconstruction and identification efficiency can be measured directly from $Z \rightarrow e^{+} e^{-}$ data using the so-called "tag and probe" method. The method consists of selecting electron candidate pairs close to $\mathrm{Z}$ mass with one electron (the tag one) passing a set of cuts which provide excellent background rejection under the Z-boson mass peak. Once the clean sample of events is selected using the tag electron, the efficiency to pass a different set of cuts for the other electron (the probe one) can be measured. $Z \rightarrow e^{+} e^{-}$events can be used also for energy scale inter-calibration of different regions of the calorimeter. It was shown that a calorimeter energy uniformity of 
$0.7 \%$ and a precision on the energy scale of $0.2 \%$ can be achieved for each $\Delta \eta \times \Delta \varphi=0.2 \times 0.4$. However it should be noted that such a precision can be achieved only if the distribution of EM calorimeter upstream material will be measured with a high accuracy using for example photon conversion process.

Conclusion. The ATLAS collaboration has developed powerful photon and electron reconstruction and identification algorithms which provide high efficiency and high rejection against the QCD jets. Early data from the ATLAS detector will be used to calibrate the EM energy scale, to align the EM calorimeter to the tracking system, to tune reconstruction algorithms and to measure the efficiencies in-situ using a tag and probe method.

\section{References}

[1] ATLAS Collaboration, G. Aad et al., The ATLAS experiment at the CERN Large Hadron Collider, 2008, JINST-3-S08003.

[2] ATLAS Collaboration, G. Aad et al., Expected performance of the ATLAS experiment, CERN-OPEN-2008-020, arXiv:0901.0512 